\title{
Daño pulmonar generado por sulfato ferroso y vitamina $C$ en embriones de ratas y crías, y regeneración posnecrótica por Petroselinum sativum (perejil)
}

\author{
Luzmila Troncoso, Emilio Guija, Felio Palomino, Marco Núñez, Gisela Oliveira, \\ Mercedes Soberón, Juana Flores \\ Centro de Investigación de Bioquímica y Nutrición, Facultad de Medicina, UNMSM
}

Objetivos: Identificar histológicamente la protección por el Petroselinum sativum (perejil) del tejido pulmonar, frente al daño por la ingesta de sulfato ferroso y vitamina $C$, en ratas madre, fetos y crías.

Diseño: Analítico, experimental.

Institución: Centro de Investigación de Bioquímica y Nutrición, Facultad de Medicina, UNMSM.

Material biológico: Ratas hembras albinas en su primera, segunda o tercera semana de preñez, fetos y crías.

Intervenciones: Se utilizó 36 ratas. Recibieron la misma dieta y agua ad líbitum y tratamientos por vía oral, durante 7 días, primera (1), segunda (2) o tercera (3) semana de preñez. Los subgrupos A1, A2, A3 recibieron sulfato ferroso (1 mg de hierro elemental) y vitamina C (10 mg); los subgrupos B1, B2, B3, sulfato ferroso (1 mg de hierro elemental), vitamina C (10 mg) y extracto acuoso de perejil (150 mg/kg); los subgrupos C1, C2, C3, agua destilada (1 mL).

Principales medidas de resultados: Hemorragia septal e intraalveolar, enfisema focal.

Resultados: El tratamiento con sulfato ferroso y vitamina C ocasionó daño pulmonar, hemorragia septal e intraalveolar moderada a severa, enfisema focal moderado; el tratamiento con sulfato ferroso, vitamina C y perejil ocasionó menos daño pulmonar y hemorragia septal leve a moderada. Las ratas sin tratamiento mostraron pulmones sin alteraciones. Hubo diferencia estadisticamente significativa entre los grupos.

Conclusiones: El tratamiento antianémico con sulfato ferroso y vitamina $\mathrm{C}$ administrado a ratas preñadas produjo daño pulmonar en ellas y en sus fetos y crías. El perejil las protegió, presentando menor daño pulmonar.

Palabras clave: Daño pulmonar, radicales libres, perejil, sulfato ferroso, vitamina C, ratas.

\section{Niveles de hemoglobina y puntajes de ingreso, en los alumnos ingresantes 2011 de la Facultad de Medicina Humana de la Universidad Nacional Mayor de San Marcos}

Luzmila Troncoso, Felio Palomino, Gisela Oliveira, Gudelia Escudero, Juana Flores, Carmen Aranda, Yorita Vargas

Centro de Investigación de Bioquímica y Nutrición, Facultad de Medicina, UNMSM

Objetivos: Determinar el nivel de hemoglobina y el puntaje de ingreso de los alumnos ingresantes 2011 de la Facultad de Medicina Humana de la UNMSM.

Diseño: Descriptivo, observacional.

Institución: Centro de Investigación de Bioquímica y Nutrición, Facultad de Medicina, UNMSM.

Participantes: Alumnos de las EAP de Enfermería y Nutrición de la Facultad de Medicina de la UNMSM.

Intervenciones: Participaron voluntariamente 45 alumnos de las EAP de Enfermería y Nutrición de la Facultad de Medicina de la UNMSM. Se les realizó la determinación de la hemoglobina en base al método de la cianometahemoglobina. Se identificó el puntaje de ingreso a la Facultad. Firmaron su consentimiento.

Principales medidas de resultados: Nivel de hemoglobina y el puntaje de ingreso.

Resultados: Los niveles de hemoglobina se encontraron dentro de los valores normales. Los alumnos de la EAP de Nutrición (16,7 g/dL) tuvieron mayores valores de hemoglobina que los alumnos de EAP de Enfermería $(15,3 \mathrm{~g} / \mathrm{dL})$. En las alumnas, no existió diferencia significativa entre la EAP de Nutrición $(13,5 \mathrm{~g} / \mathrm{dL})$ y la EAP de Enfermería $(13,7 \mathrm{~g} / \mathrm{dL})$. Los alumnos de la EAP de Nutrición (1163) tuvieron ligeramente menores valores de puntaje de ingreso que los alumnos de EAP de Enfermería (1172). En las alumnas, existió una clara diferencia significativa (EAP de Nutrición 1191 y EAP de Enfermería 1122). Existió correlación entre los niveles de hemoglobina y el puntaje de ingreso.

Conclusiones: Los niveles de hemoglobina y los puntajes de ingreso fueron mayores en los alumnos de la EAP de Nutrición, ingresantes 2011 a la UNMSM.

Palabras clave: Hemoglobina, universitarios, anemia, puntaje de ingreso, Universidad Nacional Mayor de San Marcos. 